\title{
The evolution of medical education and what scares us about it
}

\author{
I cannot teach anybody anything. I can only make them think.
}

Sócrates

In the early Middle Ages, future doctors were not students; they were apprentices. Teaching consisted of knowledge about herbs and surgical skills, "taught" by older, experienced peers. In monasteries, monks occupied themselves copying classic texts in the scriptorium. These sources, however, were not consulted by apprentices at the time. In convents and nurseries, manuals of medical botany, especially the ones about herbs of their local orchards, were preferable reading.

The first record on medical training in literature is attributed to Charaka, a Hindu physician who in 500 BC referred to the distinctive idea of a "master" from whom one could learn the art and medical practice. In the tenth century, in a somewhat pre-academic teaching style developed in Salerno and later in the Alexandrian model, Mentors were respected celebrities, authorized and of great powers. Shortly afterwards, Constantin came to Monte Cassino to translate Arabic medical writings into Greek (internationalizing medicine) and the University of Bologna was founded on September 18, 1088, by and for students.

Next, Montpellier (1289) became the Mecca of scholastic medicine, with its ambitions and methods: institutions were founded, students enrolled, and a developed pedagogy and standard methods were adopted. Being a doctor was based on static learning; they probably had books, so the transmission was therefore very easy. Dissections were made to confirm things, not to figure them out. Ex-cathedra teaching, with the authority of those knowledgeable ones who had a title, was certainly not flexible. But other didactic methods (such as the history and teaching of medicine, thanks to the rigorous studies of the ancient written documents and their transmission) were important in establishing, interpreting and editing these texts (philology). With better translations, one could read improved versions on Humanism and the Renaissance, which brought a double critical review of classic sources.

The greatest weakness of medieval medicine was that it had withdrawn itself from surgery, because of its own feature. Post-mortem dissections were no longer merely made to prove Galen's words, but to scientifically correct them. Diffusion of printed texts and drawings stimulated the birth of individual opinions. Practical studies based on Hospitals about clinical medicine were developed, new health centers concentrated in large cities and hospitals were founded, where students could find human material and as practices would have the "bedside care" now offered to many patients. Another new medical concept developed: the pathological clinical method. The barrier between surgery and medicine was dismantled once again.

The nineteenth century was the great era of Paris, London and Edinburgh, where hospital-based teaching was used through academic methods from Oxford and Cambridge. Experimental methods and specialization were fundamental to the second biological revolution and to modern scientific medicine, with new laboratories transforming the basics of teaching subjects of physiology, pathology and bacteriology. This change constituted the great German era.

Leaving a vacuum in time, for lack of space in the editorial, we extol the works of Abraham Flexner, Sir William Osler, William Welch, among others.

\section{Medical education in the twentieth century and challenges for the $21^{\text {st }}$ century}

If we look at 2020, we will recognize the need to prepare ourselves for a new generation of medical students. Known as Generation Z, Millennials come from smaller families with older parents who demonstrate traditional values and mothers who work outside their homes. Their lives were rigorously programmed, monitored and protected. They are "Digital Natives" that have brought a great impact to the school, the first generation born connected to the Internet, with tablets, Iphones and other digital media having developed the visual capacity of their brains, being thus visual forms the most effective tools for their learning process. Multimedia usage affects the ability to focus and analyze complex information. Teachers should be prepared to teach using software, hardware and digital media, and need support in order to transform their teaching styles into more effective ones.

Research has shown that the brain of Generation $\mathrm{Z}$ is structurally different from previous generations. This has nothing to do with genetics and everything to do with how we use our brains to respond to inputs in our environment. The brain of Generation $\mathrm{Z}$ becomes connected to sophisticated and complex images. As a result, brain regions responsible for visual ability are much more developed, making visual forms of learning more effective. Auditory learning (classical class, lecture and discussion) is strongly disliked by this generation. A neuropsychiatrist at Harvard Medical School calls this trend "Acquired Attention Deficit Disorder" to describe changes in the brain seen when Generation $\mathrm{Z}$ uses this technology. This can result in the inability to focus and analyze information or complex problems. It is believed that our constant bombardment of small pieces of information from Twitter, Facebook or TV with its six-second image standards are reconfiguring the brain.

Rev Med UFC. 2018;58(3):6-7. 


\section{What can we do?}

Successful teaching requires understanding and appreciation of learners' needs, origins, interests, and learning styles. In medical education today, as in most academic settings, many teachers are selected to teach based on experience and expertise. This results in a board of senior educators who were born earlier than the generations of their students. Based only on the age difference, there is significant probability of intergenerational tension between teachers and students. In search of intergenerational peacemaking, David H. Roberts and colleagues identified 12 facilitation points for Millennials teaching:

1. Educate yourself on the concept of differences between generations.

2. Recognize the environmental and cultural forces that affect the millennial student.

3. Understand how intergenerational tension can impact learning.

4. Understand that Millennials need guidance and focus on their learning.

5. Identify your teaching method or life philosophy.

6. Learn to use current e-learning technologies.

7. Recognize that Millennials value and expect aesthetically appealing (visual) educational presentations.

8. Emphasize opportunities for additional help and support.

9. Encourage modern forms of curiosity and exploration.

10. Recognize the importance of team dynamics and encourage collaboration.

11. Be fair and objective.

12. Identify the limits of multitasking.

\section{A evolução da educação médica e o que nos assusta}

Eu não posso ensinar nada a ninguém, só posso fazê-los pensar.

Sócrates

Nos primórdios da Idade Média, os futuros médicos não eram estudantes, eram aprendizes. O ensino constava de conhecimentos sobre ervas e habilidades cirúrgicas, "ensinado" por pares mais velhos. Nos mosteiros, concomitantemente, mas independentes, os monges se ocupavam copiando textos clássicos no scriptorium. Estas fontes, no entanto, não foram consultadas por aprendizes na época. Nos conventos e enfermarias preferiam manuais de botânica médica, especialmente das ervas de seus pomares locais.

O primeiro registro sobre a formação médica na literatura é atribuído a Charaka, um médico hindu que em 500 aC se referiu à idéia distintiva de um "mestre" de quem um poderia aprender a arte e a prática médica. No século 10, uma espécie de ensino pré-acadêmico desenvolvido em Salerno e mais tarde no modelo Alexandrino, os Mentores foram personalidades respeitadas, autorizadas e com grandes poderes. Logo depois, Constantin veio para Monte Cassino, traduzindo para o grego os escritos médicos árabes (internacionalizando a medicina) e a Universidade de Bolonha foi fundada em 18 de setembro de 1088 por estudantes e para estudantes.

Em seguida, Montpellier (1289) tornou-se a Meca da medicina escolástica com suas ambições e métodos: instituições foram fundadas, alunos matriculados, uma pedagogia desenvolvida e métodos padrões foram adotados. Ser médico foi baseado em um aprendizado estático, provavelmente tinham livros: a transmissão foi, portanto, muito fácil. Dissecações foram feitas para confirmar, não para descobrir. O ensino ex-cathedra, com a autoridade daqueles com conhecimento que tinham um título, foi certamente não flexível. Mas outros métodos didáticos (como a história e ensino da medicina, graças aos rigorosos estudos dos escritos antigos e sua transmissão) foram importantes no estabelecimento, interpretação e edição desses textos (filologia). Com melhores traduções, era possível a leitura de versões melhoradas sobre o Humanismo e o Renascimento, o que trouxe uma dupla revisão crítica das fontes clássicas. 
A maior fraqueza da medicina medieval era ter se separado da cirurgia, por causa de seu próprio caráter... Dissecações post-mortem não eram mais meramente feitas para provar as palavras de Galeno, mas para corrigi-las cientificamente. A difusão de textos e desenhos impressos estimulou o nascimento de opiniões individuais. Os estudos práticos baseados em Hospitais sobre a medicina clínica foram desenvolvidos, novas instituições de saúde concentradas em grandes cidades bem como hospitais foram fundados, onde os estudantes poderiam encontrar material humano, e como praticas teriam os "Cuidados de cabeceira", oferecidos agora a um grande número de pacientes. Outro novo conceito médico desenvolvido, o método clínico patológico. A barreira entre cirurgia e medicina foi desmantelada.

O século XIX foi a grande era de Paris, Londres e Edimburgo, onde o ensino baseado no hospital foi utilizado através de métodos acadêmicos de Oxford e Cambridge. Métodos experimentais e a especialização foram fundamentais para a segunda revolução biológica e para a moderna medicina científica, novos laboratórios, transformando o ensino básico das disciplinas de fisiologia, patologia e bacteriologia. Essa mudança constituiu a grande época alemã.

Com relação ao número de Lentes (leitores) dos Colégios de Medicina portugueses, deveriam ser quatro; e com a obrigação de lerem duas horas por dia para que mais facilmente se conhecessem as matérias de Medicina que haviam de ensinar. Como os cursos da Universidade deviam ser de um ano inteiro, ou pelo menos de onze meses, pouco importava que se ensinasse nas horas determinadas acima, com tanto que se ensinasse com a maior vantagem possível a favor dos Ouvintes. Se os Lentes tiverem a peito o bem público facilmente se acordarão entre si nesta direção.

Deixando um vácuo no tempo, por falta de espaço no editorial, exaltamos os trabalhos de Abraham Flexner, Sir William Osler, William Welch, entre outros.

\section{A educação médica no século XX, desafios para o século XXI}

Se olharmos para o ano de 2020, reconhecemos as necessidades de nos prepararmos para uma nova geração de estudantes de medicina, conhecida como Geração Z, eles vêm de famílias menores com pais mais velhos que têm valores tradicionais e mães que trabalham fora de casa. A vida deles foi rigorosamente programada, monitorada e protegida. São Nativos Digitais que trarão um grande impacto para a escola, é a primeira geração nascida conectado à Internet. Pelo uso da internet (tablet, Iphone, outros meios digitais) desenvolvem a capacidade visual de seus cérebros, e assim formas visuais são mais eficazes para o aprendizado desses alunos. O uso de multimídia afeta a capacidade para focar e analisar informações complexas. Os professores devem estar preparados para ensinar usando software, hardware e mídia digital, tecnológica e social. Os professores precisarão de apoio para a transformação dos seus estilos de ensinar.

Pesquisa tem mostrado que os cérebros da Geração Z são estruturalmente diferentes das gerações anteriores. Isso não tem nada com a genética e tudo para como usamos nossos cérebros para responder aos inputs em nosso ambiente. O cérebro da geração $\mathrm{Z}$ torna-se ligado a imagens sofisticadas e complexas. Como resultado, o cérebro responsável pela capacidade visual é muito mais desenvolvido, tornando as formas visuais de aprendizagem mais eficazes. Aprendizagem auditiva (aula clássica, palestra e discussão) é fortemente antipatizada por esta geração. Um neuropsiquiatra da Harvard Medical School chama essa tendência de “Transtorno do Déficit de Atenção Adquirida" para descrever mudanças no cérebro visto onde a Geração Z usa tecnologia. Isso pode resultar na incapacidade de focar e analisar informações ou problemas complexos. Acredita-se que o nosso bombardeio constante de pequenos pedaços de informações do Twitter, Facebook ou TV com seus seis segundos padrões de imagem estão reconfigurando o cérebro, espera-se que a informação seja entregue em rajadas curtas e rápidas.

\section{O que podemos fazer?}

O ensino bem sucedido requer compreensão e apreciação das necessidades, origens, interesses e dos estilos de aprendizagem dos alunos. Na educação médica de hoje, como na maioria dos ambientes acadêmicos, muitos professores são selecionados para ensinar baseados na experiência e especialização. Isso resulta em um quadro de educadores seniores que nasceram bem mais cedo que as gerações dos seus alunos. Baseado apenas na diferença de idade existe uma significativa probabilidade de tensão intergeracional entre professores e estudantes. Em busca de pacificação entre as gerações, David H. Roberts e colaboradores identificaram 12 pontos de facilitação para o ensino dos Millennials:

1. Eduque-se sobre o conceito de diferenças entre gerações.

2. Reconhecer as forças ambientais e culturais que afeta o aluno milenar.

3. Entenda como a tensão intergeracional potencial pode impactar a aprendizagem.

4. Os Milenares precisam de orientação e foco em seu aprendizado.

5. Identifique seu ensino ou filosofia de vida. 
6. Aprenda a utilizar as tecnologias atuais de e-learning.

7. Reconhecer que os Milenares valorizam e esperam apresentações educacionais esteticamente atraentes.

8. Enfatize as oportunidades de ajuda adicional, apoio e suporte.

9. Incentivar formas modernas de curiosidade e exploração.

10. Reconhecer a importância da dinâmica da equipe e incentivar a colaboração.

11. Seja justo e objetivo.

12. Identifique os limites da multitarefa.

\section{REFERÊNCIAS}

1. Silva LF, Baracat EC. Medical education - historic perspective and future challenges. Rev Med (São Paulo). 2016;95(Supl 1):28-36.

2. Roberts DH, Newman LR, Schwartzstein RM. Twelve tips for facilitating Millennials' learning. Med Teach. 2012;34(4):274-8.

3. Duin N, Sutcliffe J. A history of medicine: from prehistory to the year 2020. New York: Barnes \& Noble Books; 1992.

4. Palluault F. Medical students in Englend and France 1815-1858 [Thesis]. [Oxford]: University of Oxford; 2003.375 p.

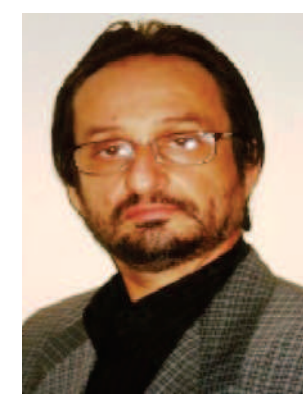

Dr Francisco das Chagas Medeiros Vice-Diretor da Faculdade de Medicina Universidade Federal do Ceará

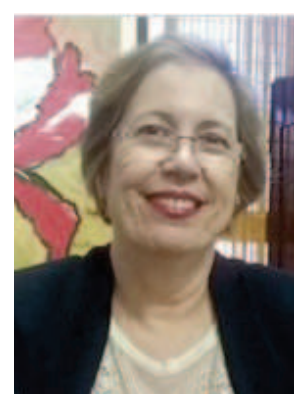

Dra Valéria Goes Ferreira Pinheiro Diretora da Faculdade de Medicina Universidade Federal do Ceará

\section{Como citar:}

Medeiros FC, Pinheiro VG. A evolução da educação médica e o que nos assusta Rev Med UFC. 2018 jul-set;58(3):6-9. 